\title{
Une modeste révision de la représentation de la théorie des systèmes d'activité
}

\author{
Robert J. Bracewell* et Jonghwi Park**
}

Résumé Le présent article propose une révision de la théorie des systèmes d'activité, qui passe de la représentation triangulaire bidimensionnelle habituelle à une représentation tétraédrique tridimensionnelle tout à fait nouvelle. Cette révision qui consiste à passer de deux à trois dimensions comporte certains avantages : elle souligne la synergie entre les divers types de médiation, ainsi que l'exigence d'une croissance réciproque pour véritablement mener à bien une activité; elle corrobore l'analyse des déséquilibres qui marquent les moyens de médiation intervenant dans l'activité; elle clarifie les relations entre les quatre champs d'activité, et permet de mieux intégrer la théorie des systèmes d'activité dans la formulation originale de l'activité de Vygotsky comme consistant dans une médiation par les outils et par les signes. La représentation tétraédrique s'annonce prometteuse sur le plan heuristique pour faire progresser la théorie et l'étude de l'activité humaine.

Mots-clés Théorie des systèmes d'activité · Médiation · Collectivité - Développement · Contradiction

\begin{abstract}
A revision of Activity Systems Theory is proposed, one that moves from the familiar twodimensional triangular representation to a novel three-dimensional tetrahedral representation. Such a revision from two to three dimensions offers a number of advantages: highlighting the synergy among meditational means, and the requirement of reciprocal growth to achieve effective activity, supporting analysis of imbalances in meditational means that interfere with activity, clarifying the relationships among the four fields of activity, and better integrating Activity Systems Theory with Vygotsky's original formulation of activity as consisting of joint sign and tool mediation. The tetrahedron representation is a promising heuristic for advancing theory and study of human activity.
\end{abstract}

Keywords Activity system theory $\cdot$ Mediation $\cdot$ Collectivity $\cdot$ Development $\cdot$ Contradiction

* Faculté d'éducation, Université McGill

** Bureau régional pour l'éducation en Asie et dans le Pacifique (UNESCO) 


\section{Une modeste révision de la représentation de la théorie des systèmes d'activité}

Cet article propose une modeste révision de la représentation triangulaire bidimensionnelle de la théorie des systèmes d'activité d'Engeström (1987). Cette révision a pour but de doter la représentation d'un pouvoir heuristique qui permette de faire progresser la théorisation et de poursuivre les études empiriques de l'activité humaine dans la perspective des systèmes d'activité.

Engeström (1987) a présenté la théorie des systèmes d'activité comme une élaboration du triangle «sujet-outil-objet » qui caractérisait la théorie de l'activité élaborée par Vygotsky (1934) et Leont'ev (1975). Dans cette élaboration, la construction matérielle de la collectivité s'ajoute aux constructions originales du sujet et de l'objet, ajout qui comporte de nouveaux types de médiation faisant appel à la collectivité, à la fois avec le sujet tel que perçu dans les règles d'interaction parmi les membres de la collectivité, et avec les objets présents dans le milieu, tels qu'observés dans les divisions du travail parmi les membres de la collectivité aux fins de produire ces objets et de travailler avec eux. En outre, l'expansion se fait d'une manière qui respecte le critère, adopté par tous les théoriciens de l'activité, et voulant que la théorie psychologique cadre non seulement avec ce que l'on voit dans la performance et le développement individuels et collectifs, mais aussi avec ce que l'on sait des éléments historiques et évolutifs du développement humain. Ainsi, Engeström amorce son élaboration théorique, non pas à partir du triangle d'activité « sujet-outil-objet », mais à partir d'un triangle présociétal «individu-population-milieu ».

Fig. 1 Représentation présociétale de l'individu, de la population et du milieu

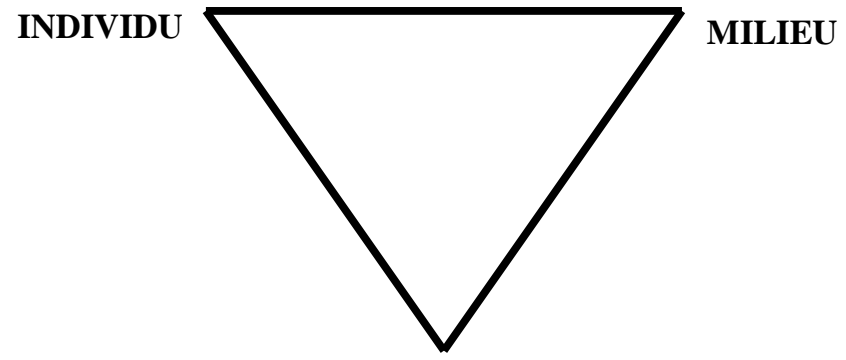

POPULATION

Engeström élabore ensuite ce triangle initial en termes de moyens de médiation qui se manifestent pour les trois types de relations naturelles (voir figure 2) :

À des niveaux supérieurs de l'évolution animale, nous constatons une rupture dans chacun des trois côtés du triangle... Le côté supérieur de la «survie individuelle » est rompu par l'émergence de l'utilisation d'outils, que les singes anthropoïdes démontrent le plus clairement... Quant au côté gauche de la «vie sociale», il est rompu par les traditions, les règles et les rites collectifs, qui prennent naissance au carrefour de l'adaptation et de l'accouplement. Enfin, le côté droit de la « survie collective » est rompu par la division du travail, influencée par les pratiques de reproduction, d'éducation et d'accouplement, et se manifestant d'abord comme la division, en cours d'évolution, du travail entre les sexes. (Engeström, 1987, p. 74-75).

Le développement complet de ces médiations se traduit par le triangle familier de la théorie des systèmes d'activité de la figure 2. Il est à noter que, dans ce processus de médiation croissante, les constructions originales du triangle proprement dites sont transformées pour passer de l'individupopulation-milieu au sujet-collectivité-objet, respectivement. 
Fig. 2 Nouvelles médiations parmi les constructions présociétales

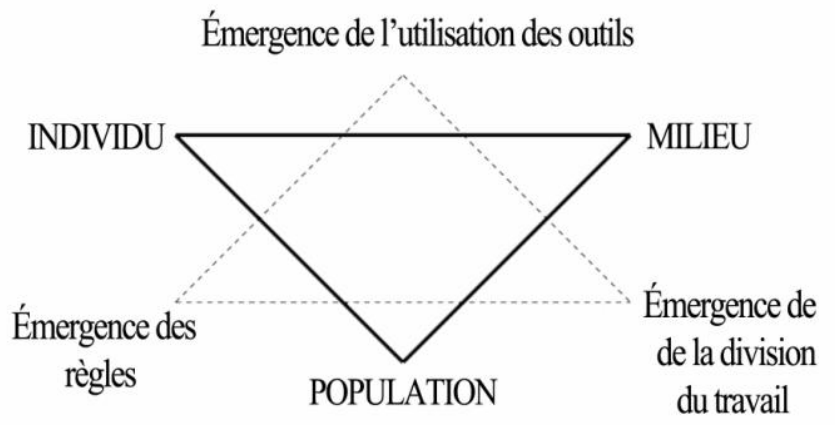

La révision que nous proposons de cette représentation concerne les constructions médiatrices des outils/symboles, des règles de communications, et de la division du travail. Elles sont présentées dans la représentation canonique de la figure 3 en tant que constructions séparées aux trois extrémités du triangle du système d'activité. Ceci est certainement compréhensible pour une représentation bidimensionnelle des constructions, mais masque l'élément commun important, à savoir que les trois sont en fait des constructions médiatrices. En effet, dans notre application de la théorie des systèmes d'activité qui consiste à explorer l'enseignement et l'apprentissage en classe (surtout dans les classes qui se servent des outils des technologies de l'information et de la communication), nous avons toujours constaté que l'emploi de ces outils est assorti de règles précises d'interaction et de division du travail parmi les participants (Bracewell et Witte, 2003, 2008; Park, 2009; Park et Bracewell, 2008; Park, Sicilia, et Bracewell, 2009). Dans notre expérience, nous avons fréquemment observé une correspondance étroite entre la division du travail et les règles de communication (ex. : une division du travail entre l'enseignant et l'élève, avec des règles de communication qui consistent dans l'enseignement et l'étude).

Fig. 3 La représentation de la théorie des systèmes d'activité (d'après Engestrom, 1987)

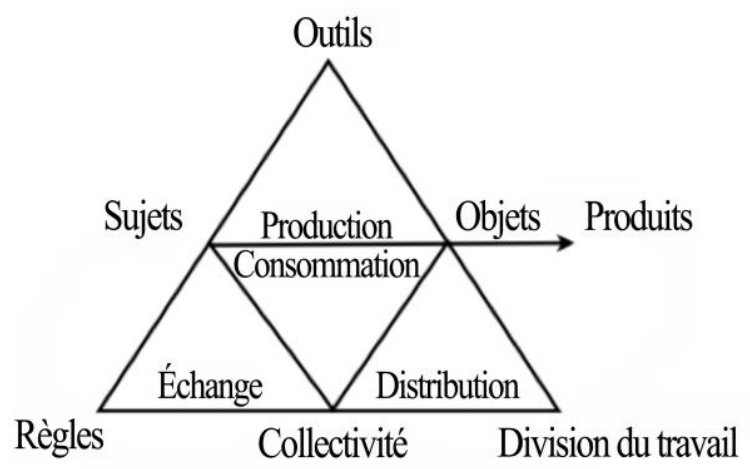

Ces éléments communs et ces correspondances de la médiation peuvent se traduire par un changement d'orientation des triangles qui formeraient ainsi un tétraèdre tridimensionnel comme l'illustre la figure 4. Dans cette figure, le triangle original «sujet-collectivité-objet » forme la base, et les trois médiateurs s'élèvent jusqu'à la pointe supérieure, tels des avions, de chaque côté du tétraèdre. 
Cette représentation tridimensionnelle comporte certains avantages heuristiques par rapport à la représentation bidimensionnelle, qui permettent de faire progresser la théorisation des systèmes d'activité et d'orienter les études empiriques de l'activité et de l'entreprise humaines.

Premièrement, elle souligne la synergie qui s'impose entre les types de médiation pour que les personnes puissent mener à bien leurs activités. L'une des analyses les plus connues de l'activité humaine qui illustre ce type de synergie est peut-être la description qu'a faite Hutchins des méthodes de navigation d'un navire, description qui comporte manifestement des instruments perfectionnés mais qui dépend de manière tout aussi évidente d'une communication complexe entre les officiers et les marins concernés, ainsi que du rôle hautement spécialisé de ces officiers et marins qui constitue la division du travail (Hutchins, 1995).

Fig. 4 Représentation tétraédrique de la théorie des systèmes d'activité (adaptée de http://en.wikipedia.org/wiki/ File:Tetrahedron.svg)

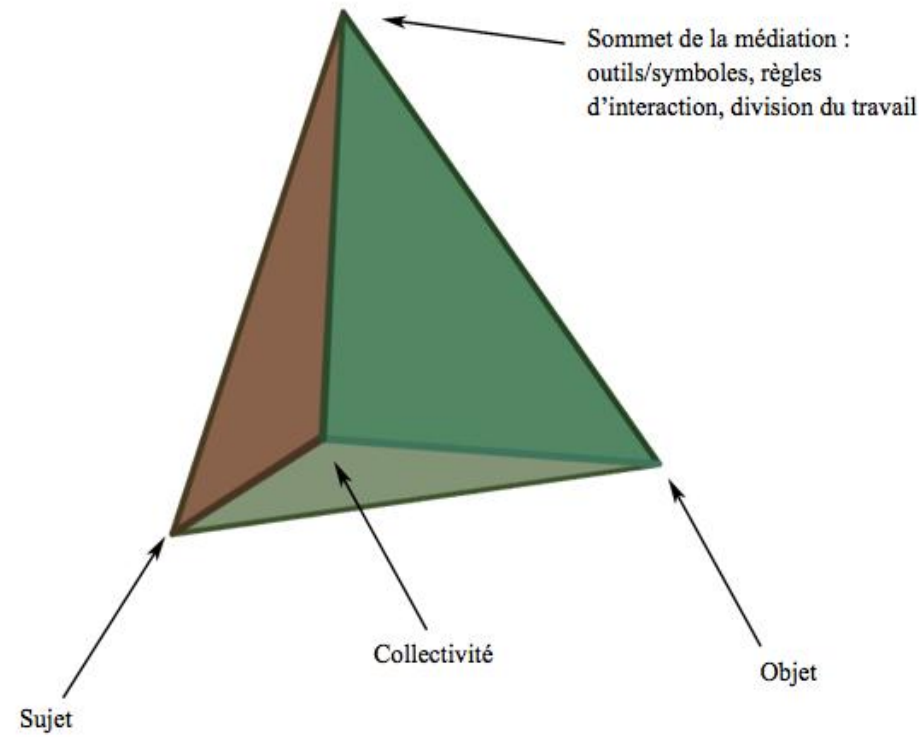

Deuxièmement, et de manière connexe, la pointe médiatrice fait ressortir une exigence de croissance (ou expansion) réciproque et proportionnée des avions qui représentent les trois types de médiation, exigence qui s'impose si l'on veut se livrer à une activité fructueuse.

Troisièmement, les avions de la médiation corroborent l'analyse des déséquilibres et des écarts qui marquent les moyens de médiation intervenant dans l'exécution réussie des activités. Dans son analyse de l'emploi des technologies de l'information en classe par l'enseignant et les élèves, Park a proposé la construction d'un système d'activité mal formé (Park, 2009, Park et Bracewell, 2008), dans lequel un ou deux des moyens médiateurs ne sont pas suffisamment déployés pour agir en synergie avec les autres moyens médiateurs. La figure 5 présente un exemple de ce type de système d'activité d'après son analyse d'une classe qui était très bien équipée en technologies de communication et d'information (c.-à-d'un ordinateur portable par élève, smartboards, communication sans fil, imprimantes), mais qui n'avait pas de règles de communication et de division du travail assez élaborées pour tirer le plus efficacement possible parti de ces technologies. 
Fig. 5 Système d'activité mal formé

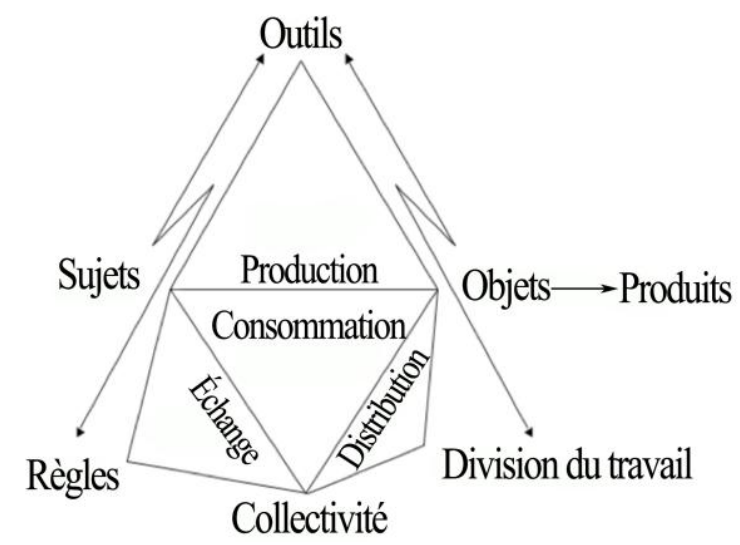

Dans cette représentation, les triangles tronqués des deux pointes inférieures du triangle du système d'activité attestent de ce manque de développement. Les flèches brisées le long des côtés du triangle représentent les tensions (contradictions) qui se manifestent dans le système d'activité et qui doivent se résoudre si l'on veut réussir à employer avec succès les technologies de communication et d'information en classe.

En ce qui concerne la représentation du tétraèdre tridimensionnel, un système d'activité mal formé est présenté à la figure 6 . Dans cette représentation particulière, la construction de la division du travail n'est pas assez achevée pour faciliter un emploi efficace des outils ou des règles de communication. Ceci se manifeste clairement dans la figure par le vide qui sépare l'avion triangulaire sur le côté antérieur, d'une part, des autres avions du tétraèdre, d'autre part, qui représentent des règles de communication et une utilisation des outils plus développées (là encore, la flèche brisée dans l'espace vide représente cette contradiction dans la capacité des constructions médiatrices). La représentation permet de voir clairement que la difficulté de résoudre la contradiction consiste à combler le vide entre les pointes des avions. Dans le meilleur des cas, ceci s'accomplirait en renforçant la division du travail parmi les participants qui cherchent à s'engager dans l'activité et à la mener à bien (dans le cas de la classe étudiée par Park, il s'agissait de doter les élèves des habiletés nécessaires pour agir plus indépendamment de l'enseignant et pour mieux apporter ensemble de l'aide sur le plan technologique).

Il importe de signaler, toutefois, que les lacunes dont souffrent les moyens de médiation peuvent être comblées de deux manières. La première consiste à renforcer ces moyens comme nous l'avons décrit ci-dessus; mais la deuxième façon consiste à réduire des moyens médiateurs mieux développés, tels que signalés précédemment, afin de les aligner sur les moyens moins achevés. Ceci semblerait être le cas pour une toute activité faisant appel aux technologies de communication et d'information en classe, où de nombreuses études ont confirmé le fait que la plupart des enseignants recourent surtout à ces technologies pour les activités d'exercisation (Becker et Riel, 2000; Ertmer, 2005; Harris Interactive, 2008; Larose et Karsenti, 2002). 
Fig. 6 Tétraèdre mal formé d'un système d'activité

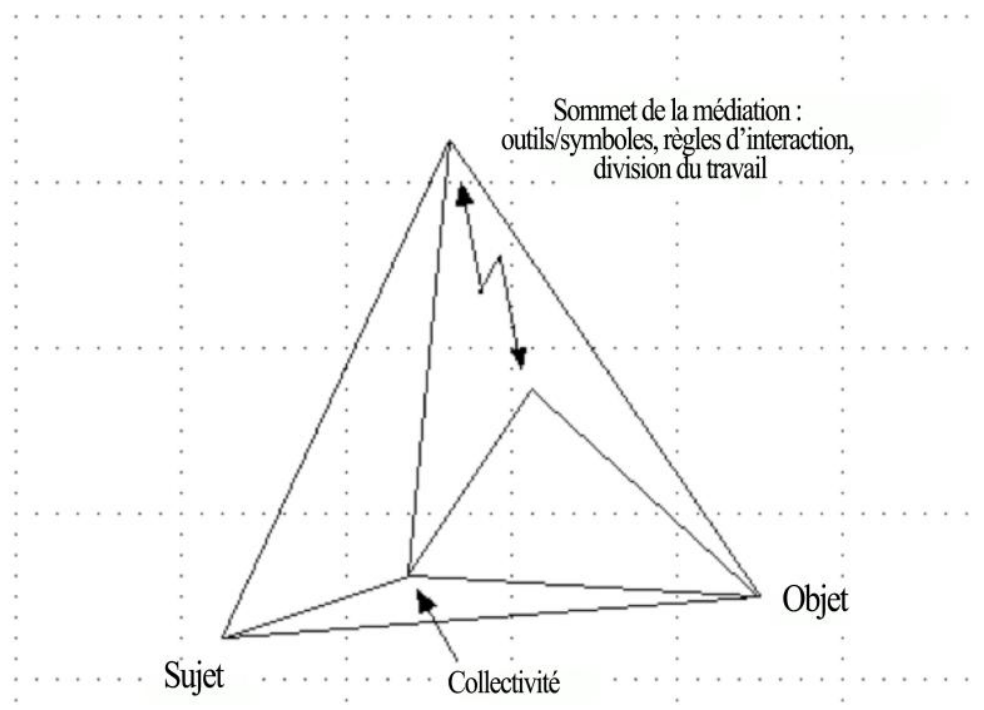

Quatrièmement, la représentation tridimensionnelle contribue à clarifier les analyses des relations parmi les constructions du système d'activité. Par exemple, dans ses recherches sur la pédagogie des sciences dans les écoles secondaires, Barma $(2008,2011)$ a démontré l'intérêt d'analyser la relation (et les contradictions) entre les constructions telles que « sujet-objet-division du travail ». Dans la représentation bidimensionnelle, cette relation peut être perçue comme un triangle entre les constructions (en d'autres termes, un triangle non canonique pour le distinguer de la présentation standard des quatre triangles apparaissant à la figure 3). Toutefois, dans la représentation tridimensionnelle, cette relation est celle qui existe le long des côtés du tétraèdre. Et, en général, la représentation tridimensionnelle accorde beaucoup plus d'importance à la relation entre les quatre champs d'activité en vertu des côtés conjoints que présentent les quatre avions.

Cinquièmement, cette révision de la forme tétraédrique permet de mieux intégrer la théorie des systèmes d'activité à la formulation originale de la médiation par les outils et par les signes de Vygotsky (Vygosky et Luria, 1994), dans laquelle l'outil ou le signe joue un rôle médiateur entre, d'une part, le stimulus tel qu'expérimenté par le sujet et, d'autre part, la réponse qui réalise un objet ou un objectif dans le milieu. Le regroupement des moyens de médiation au sommet du tétraèdre renforce la double nature matérielle et symbolique à la fois de tous les types de médiation que nous expérimentons. De toute évidence, il ne peut y avoir de séparation concrète des aspects matériels d'un outil et des moyens symboliques nécessaires à son utilisation. Un exemple historique est celui de l'astrolabe, que tout le monde reconnaît comme un outil matériel d'un certain type mais que très peu de gens savent utiliser de nos jours parce que nous ne connaissons pas les symboles qui s'y rattachent. Un exemple plus courant est la règle à calcul. Toutes les générations reconnaissent comme outil cet instrument avec ses échelles numériques, son curseur (le premier curseur, en fait), et la réglette centrale; mais seuls les membres d'une certaine génération en connaissent les symboles ou ont les connaissances voulues pour s'en servir comme calculatrice. De même, les autres formes de médiation comportent des aspects à la fois matériels et symboliques. Quant à la division du travail qui joue un rôle de médiation et qui régule l'activité de la collectivité aux fins de réaliser un objet ou un objectif, ceci se manifeste peut-être le plus clairement dans les insignes des grades militaires, même si on les trouve également dans n'importe quel domaine d'activité humaine (ex. : le casque blanc du contremaître par rapport au casque jaune de l'ouvrier). Pour ce qui concerne les règles de communication, les aspects symboliques figurent au premier plan; mais cette communication comporte toujours également un aspect matériel tel que des marques sur le papier ou des ondes sonores dans l'air. 
En conclusion, nous proposons cette représentation tridimensionnelle de la théorie des systèmes d'activité comme objet prometteur de réflexion qui permettra de poursuivre une analyse théorique fructueuse et les recherches empiriques sur l'activité humaine.

\section{Références}

Barma, S. (2008). Un contexte de renouvellement des pratiques en éducation aux sciences et aux technologies : une étude de cas réalisée sous l'angle de la théorie de l'activité. Université Laval, Québec.

Barma, S. (2011). A sociocultural reading of reform in science teaching in a secondary biology class. Cultural Studies of Science Education, 6(3), 635-661. Récupéré de : http://www.springerlink.com/content/ $\underline{59 \mathrm{n} 73 \mathrm{~m} 86 \mathrm{~g} 140978 \mathrm{~h} /}$

Becker, H. J., et Riel, M. M. (2000). Teacher professional engagement and constructivist-compatible computer use. (Report No. 7) Irvine, CA et Minneapolis, MN: Université de Californie, Irvine et Université du Minnesota: Teaching, Learning, and Computing : 1998 National Survey. Center for Research on Information Technology and Organizations.

Bracewell, R. J. et Witte, S. P. (2008). The implications of practice, activity, and semiotic theory for cognitive constructs of writing. Dans J. Albright and A. Luke (dir.), Pierre Bourdieu and Literacy Education. Mahwah (pp. 299-316). NJ: Lawrence Erlbaum Associates.

Bracewell, R. J. et Witte, S. P. (2003). Tasks, ensembles, and activity: Linkages between text production and situation of use in the workplace. Written Communication, 20, 511-559.

Engeström, Y. (1987). Learning by expanding : An activity theoretical approach to developmental research. Helsinki : Orienta-Kousultit.

Ertmer, P. A. (2005). Teacher pedagogical beliefs : The final frontier in our quest for technology integration. Educational Technology Research and Development, 53, 25-39.

Harris Interactive. (2008). The MetLife Survey of the American Teacher: Past, Present and Future. Metropolitan Life Inc.

Hutchins, E. (1995). Cognition in the Wild. Cambridge, MA : MIT Press.

Larose, F. et Karsenti, T. (2002). La place des TIC en formation initiale et continue à l'enseignement: Bilan et perspectives. Sherbrooke, Québec : Editions du CRP.

Leont'ev, A. N. (1978). Activity, consciousness, and personality (M. J. Hall, Trad.). Englewood Cliffs, NJ : Prentice-Hall. (Publication originale russe en 1975)

Park, J. (2009) Designing a well-formed activity system for an ICT-supported constructivist classroom: A Cultural-Historical Activity Theory perspective. Thèse de doctorat, Université de McGill.

Park, J. et Bracewell, R. J. (2008). Designing a well-formed activity system for an ICTs-supported classroom Dans J. Zumbach, N. Schwartz, T. Seufert, et L. Kester (Dir.), Beyond knowledge: The legacy of competence in meaningful computer-based learning environments (pp. 101-110). New York: Springer Science.

Park, J., Sicilia, C. et Bracewell, R. J. (2009). The role of contradictions in the development of technologysupported constructivist classroom practices: A cultural-historical activity theory perspective. Educational Technology International, 10(1), 1-10.

Vygotsky, L. S. (1987). Thinking and speech (N. Minick, Trans.). Dans The collected works of L. S. Vygotsky, Vol. 1: Problems of general psychology (R. W. Rieber et A. S. Carton, Dir.) (Publication originale russe en 1934 Myschlenie i rechí, pp. 39-285, 375-384, 387-389). New York : Plenum.

Vygotsky, L. S. et Luria, A. R. (1994). Tool and symbol in child development (A. R., Luria et M., Cole, Trad.). Dans R. van der Veer et J. Valsiner (Dir.), The Vygotsky reader (pp. 97-174). Oxford : Blackwell. 